\title{
Medical Image Coding Using Le Gall Transform
}

\author{
Andrey Zemtsov \\ Department of Computers and Systems \\ Volgograd State Technical University \\ Volgograd, Russian Federation \\ E-mail: ecmsys@yandex.ru
}

\begin{abstract}
The problem of approximation of medical images using wavelets is considered in the article. We propose a technique for approximating medical images using the Le Gall transform. The results of numerical experiments showing the possibility of using the technique for solving medical image processing problems are presented.
\end{abstract}

Keywords-image coding; image compression; wavelet transform; Le Gall transform; quality metrics, PSNR.

\section{INTRODUCTION}

As the development of information and telecommunication systems as well as technical means image registration, there has been an intensive growth in the application of digital imaging techniques in various areas of human activity. Increasing the requirements of end-users to such systems is one of the main reasons for their continuous improvement and development, increasing the performance and efficiency of their operation. It has now become apparent that the traditional approach to the analysis of stationary signals based on the Fourier transform is ineffective for the representation of functions and signals with local singularities [1]. One of the main disadvantages of this approach is the use as a basic function of a sinusoid having a domain of definition is $R$. As a consequence, the Fourier transform does not have a good localization in space, and is incapable of describing nonstationary signals. Indeed, for non-stationary signals, it is important to determine the times when the frequency response changes, so the basis functions must have a finite domain of definition. Some aspects of the mathematical theory of wavelets have been studied quite a long time ago. Haar proposed in 1910 a complete orthonormal system of basis functions with a local domain of definition [2]. Grossman and Morle in the work on digital processing and analysis of seismic signals [3] for the first time introduced the concept of discrete wavelet transform. However, some researchers [4] note that the technique of using shifts and scales was used by Calderon [5]. The concept of multiresolution analysis was first introduced by Meyer [6] and Malla [7] and further developed by Malla [8][9].

Today, all modern diagnostic systems in healthcare institutions are heavily related to digital medical imaging. Image slice resolutions and pixel bit-depths is constantly increasing. The most recent generation of Computed Tomography (CT) systems have 64 slices using 0.6-mm collimation can routinely resolve $0.4 \mathrm{~mm}$ objects [10]. Volumetric capable scanning technologies are increasing the amount of output data even more. Consequently, efficient medical image coding underlying improved compression and transmission techniques for handling medical images are of special importance.

Generally a large variety of existing image coding approaches using a transform based techniques utilizing discrete cosine transform [11][12], discrete wavelet transforms [13][14] or Karhunen-Loeve transforms to prediction-based techniques, such as CALIC [15] or LOCO-I [16]. Most medical systems using the Digital Imaging and Communications in Medicine (DICOM) standard [17], which based on major ISO/IEC and ITU-T standards such as JPEG [12], JPEG-LS [18] and JPEG 2000 [13]. Thus, the technique of image approximation is the main stage in the construction of a set of subsequent processing and analysis algorithms.

\section{PRINCIPLES OF DISCRETE WAVELET TRANSFORM}

We denote by $L^{2}(0,2 \pi)$ the set of all measurable functions $f$, defined on the interval $(0,2 \pi)$ and such that:

$$
\int_{0}^{2 \pi}|f(x)|^{2} d x<\infty
$$

Here $f$ is periodically piecewise on $R$ continuous function, therefore $L^{2}(0,2 \pi)$ it is called a $2 \pi$-periodic function with mean square convergence. The function $f$ can be represented by the Fourier series [19]:

$$
f(x)=\sum_{n=-\infty}^{\infty} c_{n} e^{i n x},
$$

where $c_{n}$ are the Fourier coefficients, which are defined as:

$$
c_{n}=\frac{1}{2 \pi} \int_{0}^{2 \pi} f(x) e^{-i n x} d x .
$$

It can be seen from formula (2) that in order to obtain a transformation it is necessary to have information about the signal behavior not only in the past, but also in the future [20]. Any function $f \in L^{2}(0,2 \pi)$ is represented by a superposition of integer stretches of the basis function $\omega(x)=e^{i x}=\cos (x)+i \sin (x)$ being a sinusoid. It should be noted that the function $\omega_{n}(x)=\omega(n x)$ does not belong to the space $L^{2}(R)$, which is one of the cases of a Hilbert space for which:

$$
\int_{-\infty}^{\infty}|f(x)|^{2} d x<\infty
$$

It follows from (4) that any $f \in L^{2}(R)$ must decay when $x \rightarrow \pm \infty$ and $\omega_{n}(x) \notin L^{2}(R)$. Thus, to use the functions $\omega_{n}(x)$ to generate $L^{2}(R)$ is necessary that $\omega_{n}(x)$ faded to zero when $x \rightarrow \pm \infty$. 
The basic idea of wavelet analysis is the decomposition of a signal $f$ by a basis of functions $\psi_{i}[1]$ :

$$
f=\sum_{i} c_{i} \psi_{i},
$$

i.e. in the representation of the original signal in the form of a weighted sum of basis functions $\psi_{i}$, multiplied by the spectral coefficients $c_{i}$. It follows from (5) that only spectral coefficients $c_{i}$ represent information about the original signal $f$, since the basis functions $\psi_{i}$ are assumed to be given. As noted earlier in (2), the Fourier series expansion is also represented in accordance with this concept.

To effectively represent the original signal $f$ by a small number of spectral coefficients $c_{i}$ it is necessary to require the correspondence of the basis function $\psi_{i}$ to the features of the original signal $f[20]$. Real signals are localized both in the frequency and in the time domain. The first set of signals is effectively represented by the Kronecker impulse function:

$$
\psi_{i}(t)=\delta_{i}(t)=\left\{\begin{array}{l}
1, \mathrm{k}=\mathrm{t} \\
0, \mathrm{k} \neq \mathrm{t} .
\end{array}\right.
$$

A compromise approach for signal analysis is the wavelet transform of signals based on a multiresolution analysis. In contrast to the windowed Fourier transform, the basis functions of the wavelet transform correspond to the effective time window, tuned by scaling them to the fundamental frequencies of the signal.

\section{THE MULTIRESOLUTION ANALYSIS}

A multiresolution analysis is a sequence $\left\{V_{j}\right\}_{j \in Z}$ of closed subspaces $L^{2}(R)$, satisfying the following properties:

$$
\begin{gathered}
\frac{V_{j} \subset V_{j+1} ;}{\mathrm{Y}_{j \in Z} V_{j}}=L^{2}(R) ; \\
\mathrm{I}_{j \in Z} V_{j}=\{0\} ; \\
f(x) \in V_{j} \Leftrightarrow f(2 x) \in V_{j+1} ; \\
f(x) \in V_{0} \Leftrightarrow f(x-k) \in V_{0} .
\end{gathered}
$$

There exists a function $\varphi \in V_{0}$ such that $\{\varphi(x-k)\}_{k \in Z}$ generates a Riesz basis in $V_{0}$.

A multiresolution analysis defines a sequence of disjoint nested approximation spaces $V_{j}$ in $L^{2}(R)$. If $P_{j}$ is an orthogonal projection onto $V_{j}$, then it follows from condition (8) that $\lim _{j \rightarrow \infty} P_{j} f=f$ for any function $f \in L^{2}(R)$. By (10), all subspaces $V_{j}$ are uniquely determined from the space $V_{0}$ by means of the corresponding scaling of one function $\varphi$. A function $\varphi$ is called a scaling function if it generates a multiresolution decomposition. The spaces $V_{j}$ and $V_{j+1}$ are connected by such a relation that when the scale of the function $f$ of space $V_{j}$ by $2, f$ becomes an element of the space $V_{j+1}$.

Signals represented by the square-integrable functions defined on the real axis form a Hilbert space $L^{2}(R)$ that always has orthonormal bases:

$$
\begin{gathered}
\left\langle e_{n}, e_{m}\right\rangle=\delta_{n m}, \\
\|f\|^{2}=\int|f(x)|^{2} d x=\sum_{n}\left|\left\langle f, e_{n}\right\rangle\right|^{2}
\end{gathered}
$$

In a Hilbert space there also exist more general families of linearly independent basis vectors, called the Riesz basis, for which condition (12) is generalized to two inequalities:

$$
\alpha\|f\|^{2} \leq \sum_{n}\left|\left\langle f, e_{n}\right\rangle\right|^{2} \leq \beta\|f\|^{2}
$$

where $\alpha>0, \beta<\infty$. These bases are unconditional and the order of placement in the basis of vectors can be arbitrary.

There exists a function $\varphi_{j, k}(x)=2^{j / 2} \varphi\left(2^{j} x-k\right) ; j, k \in Z$ such that, as a consequence of (10) and (11), the sequence $\left\{\varphi_{j, k}\right\}_{k \in Z}$ is a Riesz basis in $V_{j}$ for any $j \in Z$.

There is a sequence $\left\{h_{k}\right\}_{k \in Z}$ such that:

$$
\varphi(x)=\sqrt{2} \sum_{k \in Z} h_{k} \varphi(2 x-k)
$$

The expression (14) is called the scaling equation of the multiresolution analysis for the scaling functions.

The space $W_{j}$ is defined as the orthogonal complement to $V_{j}$ in $V_{j+1}$, i.e. $V_{j+1}=V_{j} \oplus W_{j}$ and $W_{j} \perp V_{j}, j \in Z$.

В силу (8) и (9) имеет место следствие:

$$
L^{2}(R)=\ldots \oplus W_{-1} \oplus W_{0} \oplus W_{1} \oplus \ldots=\oplus_{j \in Z} W_{j}
$$

Let there exist a wavelet function $\psi_{j, k}(x)=2^{j / 2} \psi\left(2^{j} x-k\right), j, k \in Z$. Then, as a consequence of (14), the sequence $\left\{\psi_{j, k}\right\}_{k \in Z}$ is a Riesz basis in $W_{j}$ for any $j \in Z$ and, there is a sequence $\left\{g_{k}=(-1)^{k} h_{1-k}\right\}_{k \in Z}$ such that:

$$
\psi(x)=\sqrt{2} \sum_{k \in Z} g_{k} \varphi(2 x-k),
$$

where $\left\{h_{k}\right\}$ is the filter coefficients for the scaling function, $\left\{g_{k}\right\}$ is the filter coefficients for the wavelet function allowing numerically to find the values $\varphi$ and $\psi$, and also the spectral coefficients of the discrete wavelet transform $\left\{d_{j}\right\}$. We refer to the equation (16) as the wavelet equation. An example of decomposition of a signal in space $R^{2}$ is shown in Fig. 1.

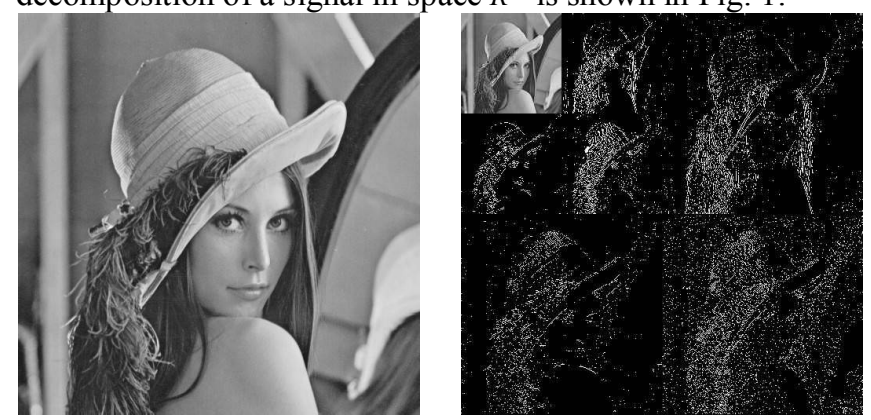

Fig. 1. An example of a two-dimensional signal and its three level wavelet transform

The wavelet transform effectively localizes low-frequency components in the frequency domain and high-frequency components in time domain, which plays a decisive role in the analysis of signals of complex shape. 
Another advantage of the wavelet transform in comparison with the Fourier transform and the discrete cosine transform is the ability to construct fast calculation algorithms.

\section{THE LE GALL TRANSFORM}

Since $V_{j}=V_{j-1} \oplus W_{j-1}$ it is possible to define a function $f(x)$ written in terms of basis functions of space $V_{j}$, in terms of basis functions of spaces $V_{j-1}$ and $W_{j-1}$ taking into account (14) and (16):

$$
\begin{gathered}
P_{j} f(x)=\sum_{k} c_{j, k} \varphi_{j, k}(x)=\sum_{k} c_{j-1, k} \varphi_{j-1, k}(x)+ \\
+\sum_{k} d_{j-1, k} \psi_{j-1, k}(x)
\end{gathered}
$$

Thus, a discrete wavelet transform is defined as a map $W$, that maps sequence $\left\{c_{j, k}\right\}$ to sequence $\left\{c_{j-1, k}, d_{j-1, k}\right\}$.

The equation (17) is called a fast wavelet transform in connection with the fact that it has complexity $O(n)$, i.e. the complexity of the calculations is proportional to the length of the signal.

It should also be noted that $\psi(x)$ from (16) is determined by the coefficients $\left\{g_{k}\right\}_{k \in Z}$, if a generating scaling function $\varphi(x)$ is defined that is determined by the coefficients $\left\{h_{k}\right\}_{k \in Z}$ of relation (14). Consequently, the system of wavelet functions and scaling functions can be defined by a set $\left\{g_{k}\right\}_{k \in Z}$ and $\left\{h_{k}\right\}_{k \in Z}$.

It follows from (17) that any function $f \in L^{2}(R)$ at some given level of resolution $j_{n}$ is representable in the form:

$$
f(x)=\sum_{k} c_{j n, k} \varphi_{j n, k}(x)+\sum_{j n} \sum_{k} d_{j, k} \psi_{j, k}(x) .
$$

It can be seen that the first term of the weighted sum (18) is a rough approximation of $f$ and $d_{j, k}$ is a detail coefficients. In other words, the resolution of the representation of some given signal increases. Note that the transition from level $j$ to level $j+1$ is equivalent to replacing it $x$ by $2 x$.

To compute the Le Galle transform [10], we use the corresponding filter coefficients for the scaling and wavelet functions:

$$
h_{k}=\left\{-\frac{1}{8},-\frac{1}{4}, \frac{3}{4},-\frac{1}{4},-\frac{1}{8}\right\}, g_{k}=\left\{\frac{1}{2}, 1, \frac{1}{2}\right\}
$$

Taking into account (18) and (19), we find expressions for the decomposition of the signal in the form:

$$
\begin{gathered}
d_{j, k}=c_{j-1,2 k+1}-\frac{1}{2}\left(c_{j-1,2 k}+c_{j-1,2 k+2}\right), \\
c_{j, k}=c_{j-1,2 k}+\frac{1}{4}\left(d_{j, k-1}+d_{j, k}\right) .
\end{gathered}
$$

Reconstruction is carried out in the reverse order:

$$
\begin{gathered}
c_{j-1,2 k}=c_{j, k}-\frac{1}{4}\left(d_{j, k-1}+d_{j, k}\right), \\
c_{j-1,2 k+1}=d_{j, k}+\frac{1}{2}\left(c_{j-1,2 k}+c_{j-1,2 k+2}\right) .
\end{gathered}
$$

It should be noted that $h_{k}$ and $g_{k}$ in (19) are also coefficients of the form $c \cdot 2^{i}$ where $c, i \in Z$. Consequently, in computing the Le Gall transform a computationally complex multiplication operation can be replaced by a shift by $i$ bit.

\section{THE NUMERICAL EXPERIMENTS}

Let us demonstrate with a concrete example the possibility of applying the proposed method of approximation of medical images. For comparison of various algorithms, the characteristic of the degree of image distortion is often used. To estimate the distortion, we will use the measure of the Peak Signal-to-Noise Ratio (PSNR). This visual quality assessment index is generally accepted in the given subject area and is computed by the following equation:

$$
P S N R=20 \times \log _{10} \frac{255}{\sqrt{\frac{1}{M \times N} \sum_{j=1}^{N} \sum_{i=1}^{M}\left(f_{i, j}-g_{i, j}\right)^{2}}},
$$

where $M$ and $N$ are the image width and height namely; $f$ and $g$ are the original and reconstructed component values namely.

The degree of distortion measured through the ratio of the signal level to the noise level is determined by the reconstruction of the signal, and the comparison of the result signal with the source signal. To estimate the volume of the reconstructed images, we will also estimate the specific number of bits (bpp, bit per pixel) needed to store the reconstructed image. As a test image we will use a brain scan using 64-slice spiral CT. Fig. 2 shows the CT images reconstruction using Haar wavelets for various low bitrates such as $0.4 \mathrm{bpp}, 0.1 \mathrm{bpp}, 0.04 \mathrm{bpp}$ and $0.01 \mathrm{bpp}$.

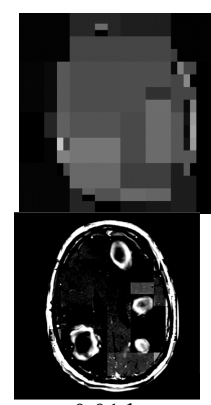

$0.01 \mathrm{bpp}$

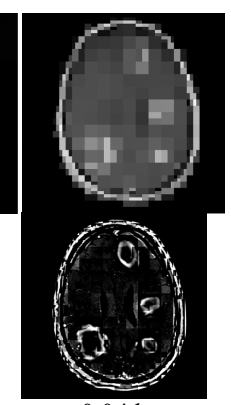

$0.04 \mathrm{bpp}$

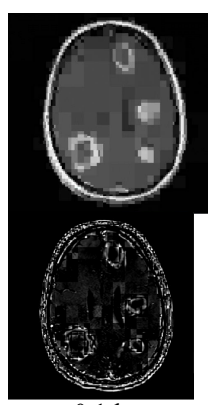

$0.1 \mathrm{bpp}$

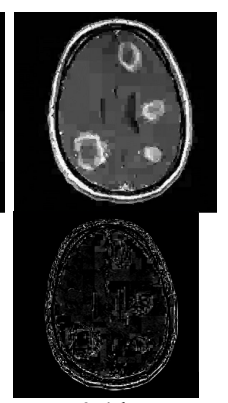

$0.4 \mathrm{bpp}$
Fig. 2. Reconstructed CT images using Haar wavelets

The recovered images can not be considered satisfying the information requirements for medical images. One of the drawbacks of Haar wavelets hindering their wide application is the lack of smoothness, which is evident in the presence of the blocking artifact.

Fig. 3. shows the visual results performed by proposed method for various low bitrates. These images shows a pixel error, i.e. the degree of distortion added by the proposed method into the CT image. These results are much better than the results obtained using Haar wavelets or discrete cosine transform. It should be noted that for quantifying most pathologies these bitrates are not suitable. 


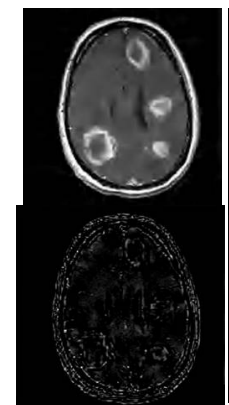

$0.01 \mathrm{bpp}$

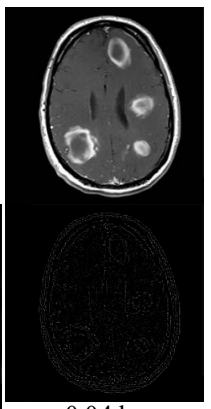

$0.04 \mathrm{bpp}$

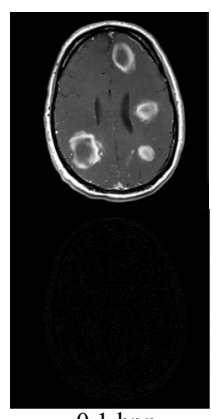

0.1 bpp

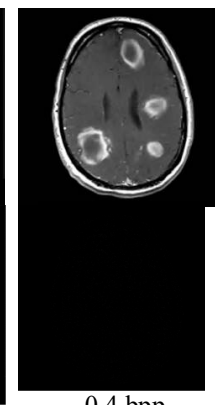

$0.4 \mathrm{bpp}$
Fig. 3. Reconstructed CT images using proposed method

Fig. 4 shows the variation of PSNR in decibels calculated for the CT images set with respect to bitrate. The difference in compression performance by calculating the image quality is presented on two rate-distortion curves.

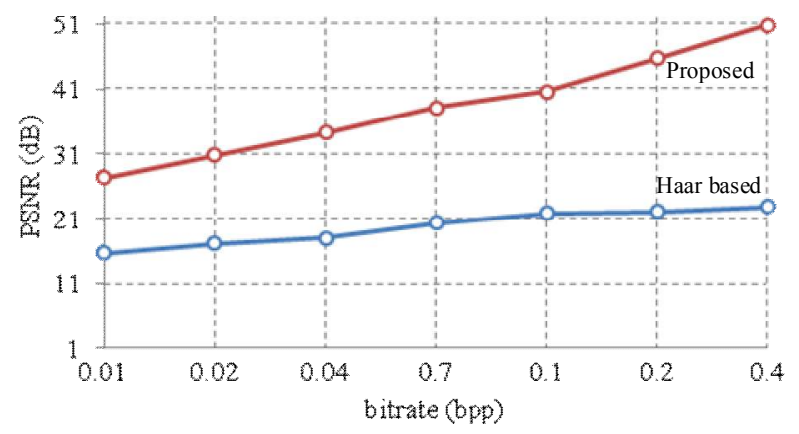

Fig. 4. Comparison on PSNR metric of proposed technique with Haar based approach at range of low bitrates.

Moreover, objects of interest are often not well contrasted against the background. The proposed method will enable more accurate quantification of object of interest, for example, quantification of cortical bone thickness for assessing bone quality in osteoporotic patients.

\section{CONCLUSION}

In this paper, we presented an effective approach to encoding medical images that is based on the basis set of forward transform coefficients using integer algebra. The proposed method of approximation of images using Le Gall transform is the main stage in the construction of a set of subsequent algorithms for image analysis and processing. Above the image represented in the form of a set of wavelet coefficients of the Le Gall transform can be perform filtering operations, for example, to remove noise, extraction of various features, thresholding, and others. The proposed approach to computing a Le Gall transform is characterized by low computational complexity. To demonstrate the possibility of applying the proposed method, the work presents the results of numerical experiments showing the possibility of using a proposed method for solving medical images processing problems. The level of detail is very high while preserving critical information that makes the method suitable for medical and telemetric imaging applications.

\section{REFERENCES}

[1] I. Daubechies, The Wavelet Transform, Time-Frequency Localization and Signal Analysis, IEEE Transactions on Information Theory, vol. 36, Issue 5, pp. 961-1005, September 1990.

[2] A. Haar, Zur Theorie der orthogonalen Funktionensysteme, Math. Ann. 69, pp. 331-371, 1910.

[3] A. Grossmann, J. Morlet, Decomposition of Hardy functions into square integrable wavelets of constant shape, SIAM J. Math. Anal. 15, pp.723736,1984

[4] C. K. Chui, An Introduction to Wavelets, Academic Press, NY, 1992 $266 \mathrm{p}$.

[5] A.P. Calderon, Intermediate spaces and interpolation, the complex method, Studia Mathematica, vol. 24, pp. 113-190, 1964.

[6] Y Meyer, Ondelettes et functions splines, Seminaire EDP, Ecole Polytechnique, Paris, 1986

[7] S. Mallat, Multiresolution representation and wavelets: $\mathrm{PhD}$ thesis, Univ. of Pennsylvania, Philadelphia, 1988.

[8] S. Mallat, A theory of multiresolution signal decomposition: the wavelet representation, IEEE Trans. Pattern Anal. Machine Intell. 11, pp. 674693, 1989.

[9] S. Mallat, Multiresolution approximations and wavelets orthonormal bases of $L^{2}(R)$, Trans. Amer. Math. Soc. 315, pp. 69-87, 1989.

[10] L.W. Goldman, Principles of CT: multislice CT, J. Nucl. Med. Technol. 36, 2008.

[11] W.B. Pennebaker, J.L. Mitchell, JPEG Still Image Data Compression Standard, 1 st edition, Kluwer Academic Publishers, 1992.

[12] ISO/IEC 10918-1 j ITU-T Rec. T.81, Information Technology - Digital Compression and Coding of Continuous-tone Still Images, 1992.

[13] ISO/IEC 15444-1 j ITU-T Rec. T.800, Information Technology - JPEG 2000 Image Coding System: Core Coding System, 2002.

[14] A.N. Zemtsov, S.Md. Rahman, Copyright protection using discrete wavelet transform, Izvestia VSTU, vol. 6, pp. 134-136, 2009.

[15] X. Wu, N. Memon, Context-based, adaptive, lossless image coding, IEEE Trans. Commun. 45, pp. 437-444, 1997.

[16] M.J. Weinberger, G. Seroussi, G. Sapiro, The LOCO-I lossless image compression algorithm: principles and standardization into JPEG-LS, IEEE Trans. Image Process. 9, pp. 1309-1324, 2000.

[17] DICOM, Digital Imaging and Communications in Medicine, URL: http://medical.nema.org, 2013.

[18] ISO/IEC 14495-1 j ITU-T Rec. T.87, Information Technology Lossless and Near-lossless Compression of Continuous-tone Still Images: Baseline, 1998.

[19] Akansu A. Wavelet, Subband and Block Transforms in Communications and Multimedia. Springer, 2010, $432 \mathrm{p}$.

[20] Zemtsov A.N, Comparative analysis of compression methods based on orthogonal expansions. Information Technology. Radioelectronics. Telecommunications, vol. 2, pp. 160-164, 2012.

[21] D. Gall, A. Tabiatai, Sub-band coding of digital images using symmetric short kernel filters and arithmetic coding techniques, Speech and Signal Processing, pp. 761-764, 1988. 